\title{
Determining the Shortest Path of Multi-location Path Based on the Maximum Flow
}

\author{
Xueying $\mathrm{Li}^{1, \text { a }}$, Qin Zhang ${ }^{1, \mathrm{~b}}$, Qiang Zhang ${ }_{\text {Yang } \mathrm{Yu}^{1, \mathrm{f}}}$, Wenxia $\mathrm{Yu}^{2, \mathrm{~d}}$ Mengzhu Shi ${ }^{1, \mathrm{e}}$, \\ ${ }^{1}$ School of Mechanical Engineering, Shandong University, Jinan 250061, China \\ ${ }^{2}$ Shandong Labor Vocational and Technical College

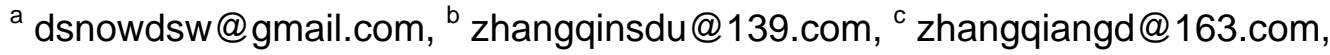 \\ d seki8827@gmail.com, ${ }^{\text {e}} 1874771425 @ q q . c o m,{ }^{f}$ 251963765@qq.com
}

Keywords: location path; shortest path; the maximum flow

\begin{abstract}
In this paper, multi-location path math model is established at the condition that the number of distribution centers, customers, and the service scope is known. Constraints are including customer demand, network matching, and vehicle loading. To solve the shortest path based on the maximum flow problem, the network model about one distribution center and six distribution customers is proposed and the solution is also given below. When the distribution of prices, vehicle specifications and node path are known, the optimal path is gotten according to check the model instance. So, it's feasible to solve the optimal path using the maximum flow.
\end{abstract}

\section{Introduction}

In the decision-making process, the diversity of vehicles is needed to be focused when designing the supply chain network. On the periodic location path problem, if manufactures could provide the stable service frequency, they can choose the service type and temporal pattern. However, when using just-in-time, this path decision is not acceptable. Therefore, the paper is proposed an approximate multi-location model to avoid the complex computation during the process.

There are two different ways to solve the location path problem in supply chain network system, the exact algorithms and heuristic algorithms. Different types of logistic network have different solving methods. Recently, heuristic algorithms use much better, such as genetic algorithm and tabu algorithm [1]. In addition, Dorigo etc. [2, 3, 4] proposed the ant colony algorithm based on population simulations to solve the traveling salesman problem. In 2005, Shuren Zhu designed a genetic algorithm based on natural numbers encoding, according to roulette copy and maximum cross-law [5].

\section{Establishing model.}

\section{Parameter variable definition}

$T$ : Planning period, $t \in\{1,2, \cdots,\|T\|\}$.

$t$ : path decision time in planning period $\mathrm{T}, t \in T$.

l: location decision time in planning period $\mathrm{T}, l \in T_{L} \subseteq T$.

I: facilities parameters in each node (vehicle types, numbers).

$J$ : customer service parameters (customer numbers, demands).

$\sigma(l)$ : the time in $T_{L}$ when $l \succ 1$.

$\tau(l)$ : the time after $l \prec \bar{l}$.

$f_{i}^{l}$ : setting costs about facility $i$, when $l \in T_{L}, i \in I$. 
$\varphi_{i}^{l}$ : maintain costs about facility $\mathrm{i}, \quad l \in T_{L} \backslash\{1\}$.

$y_{i}^{l}=\left\{\begin{array}{l}1 \\ 0\end{array}\right.$ : the punishment costs when closing facility i, $l \in T_{L} \backslash\{1\}$.

Transportation cost between $\mathrm{r}$ and $\mathrm{s}$ is defined $C_{e}^{t}, r, s \in I \cup J, r \neq s$.

$y_{i}^{l}=\left\{\begin{array}{l}1 \\ 0\end{array}\right.$ if facility $\mathrm{i}$ is opened at period $l \in T_{L}$

others

$W_{i}^{l}=\left\{\begin{array}{lr}1 & \text { if facility } i \text { is opened at period } l \in T_{L} \\ 0 & \text { others }\end{array}\right.$

For $^{i \in I, j \in J, t \in T:} \boldsymbol{Z}_{i j}^{t}=\left\{\begin{array}{cc}1 & \text { if facility } i \text { is opened at period } l \in T_{L} \\ 0 & \text { others }\end{array}\right.$

\section{Constraints}

$$
\begin{aligned}
& x^{t}(\delta(i))=2\left(y_{i}^{l}+w_{i}^{l}\right), \\
& x^{t}(\delta(j))=2, \\
& x^{t}(\delta(j))=0, \\
& x^{t}(\delta(S)) \geq 2, \\
& X_{r s}^{t} \leq Z_{r s}^{t}, \\
& X_{r s}^{t}+Z_{i_{1} r}^{t}+Z_{i_{2} r}^{t} \leq 2, \\
& \sum_{i \in I}\left(y_{i}^{l}+w_{i}^{l}\right) \geq p^{l}, \\
& \sum_{i \in I} Z_{i j}^{t}=1 \\
& y_{i}^{l}+w_{i}^{l} \leq 1 \\
& W_{i}^{l} \leq y_{i}^{\sigma(l)}+w_{i}^{\sigma(l)}, \\
& Z_{i j}^{t} \leq y_{i}^{l}+w_{i}^{l}, \\
& y_{i}^{l}, W_{i}^{l} \in\{0,1\} \\
& Z_{i j}^{t} \in\{0,1\}
\end{aligned}
$$

\section{Model description}

The time zone of the location path needs to be measured in different scope. Particularly, with the different location decisions made in time each zone of subset, the strategic path decision has to be made unique each time.

Moreover, the universality of the research appears under some special circumstances. For example, when in the plan period $T_{L}=1$, there is a single location decision. If $T_{L}=T$, the location decision is made in each time region. In this time, the orientation and path of the time are same.

To the given time zone $e^{l \in T_{L}}$, if the service is not proposed to customer, the facility would be closed. We assumed that the state of the facilities were close before the prior of the time zone. When $l \succ 1$ and the facilities serve customer normally, we call the changed position in $\sigma(l)$ opened 
state. Otherwise, call operating state. Because the location decision is made in time $T_{L}$, all the facilities can't be changed in $t \in[l, \tau(l-1)]$ to each $l \in T_{L}$.

In time $t \in T$, customers' demands $J^{T}$ are served. When ended, the beginnings and the ends are decided. However, this process needs to meet the strategic requirements that the minimum number $p^{l}$ meet the service demands in ${ }^{t} \in T_{L}$. This is to meet the customers' implicit demands. This can be also companies' strategic decision for the balance of markets. Therefore, it includes the situation of no constraints $p^{l}=0$.

\section{Model solving based on the maximum flow.}

\section{Steps}

1)Determine the client nodes needed to be served and mark.

2)For the marked client nodes, identify the unmarked nodes to which are directly connected.

3)Select the shortest path between the unmarked nodes and the marked nodes.

4)In the third step, if the selected unmarked node is the endpoint, calculation should be end. Otherwise, continue the second step.

\section{Solving}

There is a simple network distribution of solar company including six distribution points. The distance between the points is shown in Fig.1. The distribution price is known, the length of the vehicle is $12.5 \mathrm{~m}$, and the vehicles go through the distribution points.

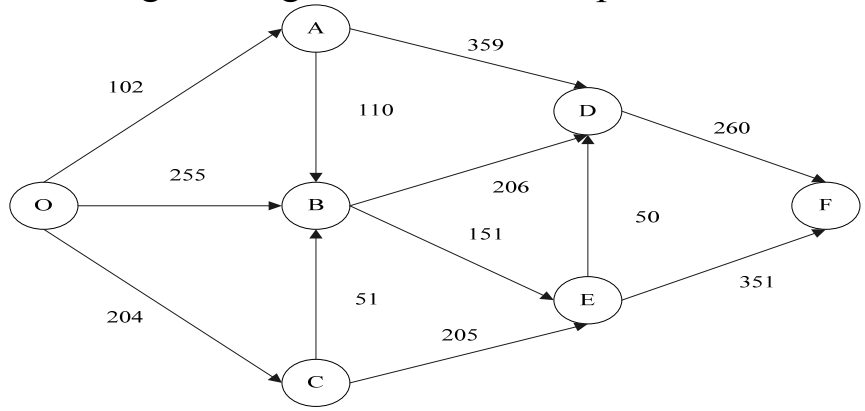

Fig.1 One distribution center and six distribution points

According to the maximum flow, the data in Table.1 can be gotten by simple calculation. In the calculation process, the distance between the tag nodes and other connected nodes is compared, and then the nearest node is chosen to the next tag node, and so on, until reach the last target F.

Tab. 1 the maximum flow

\begin{tabular}{|c|c|c|c|c|c|c|}
\hline No. & Tag point & Unmarked & distance & Nearest & Nearest & connect \\
\hline \multirow{3}{*}{1} & \multirow{3}{*}{$\mathrm{O}$} & A & 102 & \multirow{3}{*}{ A } & \multirow{3}{*}{102} & \multirow{3}{*}{ OA } \\
\hline & & B & 255 & & & \\
\hline & & $\mathrm{C}$ & 204 & & & \\
\hline \multirow{4}{*}{2} & \multirow{2}{*}{$\mathrm{O}$} & B & 255 & \multirow{4}{*}{$\mathrm{C}$} & \multirow{4}{*}{204} & \multirow{4}{*}{$\mathrm{OC}$} \\
\hline & & $\mathrm{C}$ & 204 & & & \\
\hline & \multirow{2}{*}{ A } & B & 212 & & & \\
\hline & & D & 461 & & & \\
\hline \multirow{4}{*}{3} & \multirow[t]{2}{*}{ A } & B & 212 & \multirow{4}{*}{ B } & \multirow{4}{*}{212} & \multirow{4}{*}{$\mathrm{AB}$} \\
\hline & & $\mathrm{D}$ & 461 & & & \\
\hline & \multirow{2}{*}{$\mathrm{C}$} & B & 255 & & & \\
\hline & & $\mathrm{E}$ & 409 & & & \\
\hline \multirow{2}{*}{4} & A & D & 461 & \multirow{2}{*}{ E } & \multirow{2}{*}{363} & \multirow{2}{*}{$\mathrm{BE}$} \\
\hline & B & D & 418 & & & \\
\hline
\end{tabular}




\begin{tabular}{|c|c|c|c|c|c|c|}
\hline & & $\mathrm{E}$ & 363 & & & \\
\cline { 2 - 5 } & $\mathrm{C}$ & $\mathrm{E}$ & 409 & & & \\
\hline \multirow{2}{*}{5} & \multirow{2}{*}{$\mathrm{E}$} & $\mathrm{D}$ & 413 & $\mathrm{~N}$ & 413 & \multirow{2}{*}{ ED } \\
\cline { 2 - 7 } & & $\mathrm{F}$ & 714 & & 673 & $\mathrm{DF}$ \\
\hline 6 & $\mathrm{D}$ & $\mathrm{F}$ & 673 & $\mathrm{~F}$ & 673 \\
\hline
\end{tabular}

From Tab.1, the path which is based on the maximum flow is $\mathrm{O} \rightarrow \mathrm{A} \rightarrow \mathrm{B} \rightarrow \mathrm{E} \rightarrow \mathrm{D} \rightarrow \mathrm{F}$.

\section{Conclusion}

To consider the diversity of the vehicle, the paper derives the multi-location path problem from the normal location path problem. The location path model is given, and it is analyzed in detail. Then, the maximum flow method solving the nearest path and solution steps are proposed. Last, the validation of the example is provided.

\section{References}

[1] Xu Ning, Li Chunguang, Zhang Jian, A Comparative Study of Several Modern Optimization Algorithm[J].Systems Engineering and Electronics, 2002, 24(12): 101-104.

[2] DORIGO M, MANIEZZO V, COLNMI A. Ant system: optimization by a colony of cooperating agents [J].IEEE Transactions on Systems, Man, and Cybernetics-part B·1996, 26(1): 29-41.

[3] MANIEZZO V, CARBONARO A. Ant colony optimization: an overview[c]//C Ribeiro. Essays and Surveys in Metaheuristics. [S.I.]: Kluwer, 2001:21-44.

[4] Manoj K.Malhotra. Defining the concept of supply chain quality management and its relevance to academic and industrial practice, 2005(3)

[5] Zhu Shuren, Li Wenbin, Kuang Fangjun.An optimization genetic algorithm to logistics distribution with soft time window [J].Computer Engineering and Electronics, 2005, 27(12): 108-110.

[6] Saunders, Malcolm. Strategic Purchasing and Supply chain Management, Pitman Publishing, 1994 